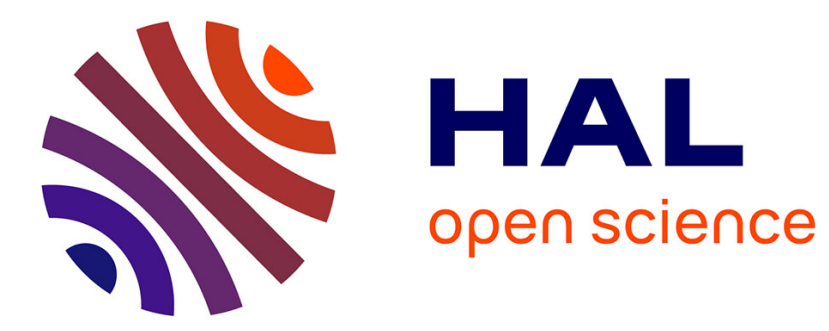

\title{
Laue diffraction lenses for astrophysics: Theoretical concepts
}

\author{
H. Halloin, P. Bastie
}

\section{To cite this version:}

H. Halloin, P. Bastie. Laue diffraction lenses for astrophysics: Theoretical concepts. Experimental Astronomy, 2006, 20, pp.151-170. 10.1007/s10686-006-9064-z . in2p3-00130755

HAL Id: in2p3-00130755

https://hal.in2p3.fr/in2p3-00130755

Submitted on 13 Feb 2007

HAL is a multi-disciplinary open access archive for the deposit and dissemination of scientific research documents, whether they are published or not. The documents may come from teaching and research institutions in France or abroad, or from public or private research centers.
L'archive ouverte pluridisciplinaire HAL, est destinée au dépôt et à la diffusion de documents scientifiques de niveau recherche, publiés ou non, émanant des établissements d'enseignement et de recherche français ou étrangers, des laboratoires publics ou privés. 


\title{
Laue diffraction lenses for astrophysics : theoretical concepts
}

Received: date / Accepted: date

\begin{abstract}
Beyond the present technologies, Laue diffraction lenses are very promising tools in the field of gamma-ray astrophysics. The theoretical concepts of this kind of instruments are based on the Laue diffraction in crystals, discovered almost 100 years ago. Though they are commonly used in crystallography, their application to $\gamma$-ray focusing in astrophysics requires some specific developments, e.g. in terms of energy and imaging responses. The present article describes the physics of X-ray diffraction in crystals. In the context of the Darwin model of mosaic crystals, some peculiar aspects, relevant to the astrophysical observation, are discussed. The evaluation and optimization of diffraction efficiency are discussed, especially w.r.t the crystal's mosaicity and thickness, its spatial extent and deviations to the "ideally imperfect" Darwin model.
\end{abstract}

Keywords Focusing optics - Gamma-ray astrophysics - Crystal diffraction

PACS 95.55.Ka $\cdot 61.50 . \mathrm{Ah} \cdot 61.10 .-\mathrm{i} \cdot 41.50 .+\mathrm{h}$

\section{Introduction}

Almost 100 years ago, Max von Laue discovered and demonstrated the diffraction of X-rays in crystals $[1,2]$. The laws of the so-called Laue (or Bragg)

\author{
Hubert Halloin \\ CESR, 9, avenue du Colonel Roche, 31028 Toulouse, FRANCE \\ Present address: APC, 11, place Marcelin Berthelot, 75231 Paris Cedex 05, \\ FRANCE \\ Tel.: +33144271533 \\ Fax: +33143546989 \\ E-mail: hubert.halloin@apc.univ-paris7.fr \\ Pierre Bastie \\ Laboratoire de Spectrométrie Physique, BP 8738402 S $^{\text {t }}$ Martin d'Hères Cedex, \\ FRANCE \\ ILL, BP 156, 38402 Grenoble Cedex 9, FRANCE
}




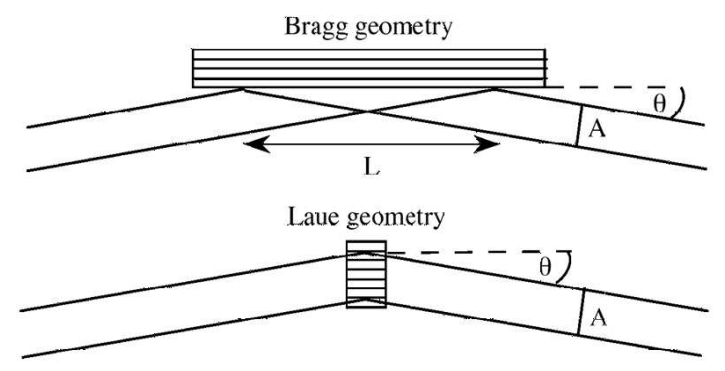

Fig. 1 Diffraction geometries

diffraction in crystals are widely used in crystallography and solid states physics. Nevertheless, their application in astrophysics is much more recent and appears as one of the most promising way to perform the sensitivity leap needed in the hard $\mathrm{X}$ - and gamma-ray regimes.

In the present paper, we will describe the bases of X-ray diffraction in crystals, from a "practical" point of view in the aim of lens design for astrophysics. The theoretical predictions presented here are used to design and study the instrumental response of a crystal diffraction lens, as described in an accompanying article ([3], this volume).

\section{Diffraction lens geometry.}

Since the wavelength of $\gamma$-ray photons is smaller than distances between atoms in solid matter, coherently deviating $\gamma$-rays has long been considered as impossible in nuclear astrophysics and only the corpuscular properties of these photons were used. Nevertheless, Friedrich, Knipping and Laue [1, 2], at the beginning of the $20^{t h}$ century demonstrated that $\gamma$-rays can interact coherently in a crystal lattice, provided that the angles of incident photons are very small and satisfy the Bragg relation :

$$
2 d \sin \theta=n \lambda
$$

where $d$ is the crystal plane spacing, $\theta$ the incident angle with respect to the crystal planes, $n$ is the reflection order and $\lambda$ the wavelength of the photon. Thus, by mounting and tuning (i.e. orienting the crystal to get the correct scattering angle $\theta$ ) adequate crystals on concentric rings, a parallel beam can be focused on a single point.

Historically, the idea of focusing X-rays through the use of crystal diffraction seems to have first been proposed by Gouy in 1915 [4]. A few years later, Dardord in 1922 [5] and Fermi in 1923 [6] independently obtained images with monochromatic X-ray beams, following the method suggested by Gouy.

The diffraction inside the crystal can either occur near the surface (Bragg geometry) or "in volume", while the beam is propagating through the entire crystal (Laue geometry, see Fig. 1). The Bragg geometry has been successfully used in multilayer optics in the X-ray regime. On the contrary, above a few hundreds of $\mathrm{keV}$, the required incident angle (given by eq. 1 ) is so small that 
the Bragg geometry appears out of question. As an example, a $1 \mathrm{x} 1 \mathrm{~cm}^{2}$ beam at $511 \mathrm{keV}$, diffracted in a Ge[111] lattice, would require a $2.8 \mathrm{~m}$ long crystal. Beyond the difficulty of producing such big crystals, the complexity of the mounting and the mass of the tiles reject the Bragg geometry as a feasible option. Eventually, the mean free path of $\gamma$-rays in crystal is usually bigger than the optimal diffraction length, and the Laue geometry can be used in a $\gamma$-ray lens design.

In the present article, we will describe the basics of the theory of X-ray diffraction in crystals, assuming a Laue geometry. The following concepts and equations are mainly taken from $[7,8,9,10]$. Please refer to these documents for further details as well as to other articles in this volume for a good coverage of recent works on hard X-ray and gamma-ray focusing, both experimental and theoretical.

\section{Diffraction by a single crystal}

Let us consider the reflection of an X-ray beam on a given plane of a crystal. $\mathbf{k}_{0}$, resp. $\mathbf{k}_{1}$, are the incident, resp. diffracted, wave vectors. With these definitions, and $\Delta \mathbf{k}=\mathbf{k}_{1}-\mathbf{k}_{0}$, it immediately follows :

$$
\Delta k=|\Delta \mathbf{k}|=2 k_{0} \sin \theta_{i}=4 \pi \frac{\sin \theta_{i}}{\lambda_{0}}
$$

where $\lambda_{0}$ is the wavelength of the incident beam, and $\theta_{i}$ is the angle of $k_{0}$ w.r.t. the plane orthogonal to $\Delta \mathbf{k}$.

The main property of a crystal is the periodicity of the elementary cell along the lattice axes $\mathbf{a}, \mathbf{b}$ and $\mathbf{c}$. Due to this periodicity, the intensity of the diffused, coherent emission is proportional to $\left(\sin N_{p} \frac{\Delta \mathbf{k}}{2} \mathbf{a} / \sin \frac{\Delta \mathbf{k}}{2} \mathbf{a}\right)^{2}$, where $N_{p}$ is the number of cells along axis a. Thus, the intensity of the diffracted beam is essentially zero unless :

$$
\boldsymbol{\Delta} \mathbf{k} \cdot \mathbf{a}=2 h \pi, \quad h \in \mathbb{Z}
$$

and similarly, considering the axes $\mathbf{b}$ and $\mathbf{c}$ :

$$
\boldsymbol{\Delta} \mathbf{k} \cdot \mathbf{b}=2 k \pi, \quad \boldsymbol{\Delta} \mathbf{k} \cdot \mathbf{c}=2 l \pi, \quad k, l \in \mathbb{Z}
$$

This set of equations forms the three Laue conditions that should be simultaneously satisfied [2]. From these equations $\Delta \mathbf{k}$ is then orthogonal to the crystal plane with Miller indexes $(h, k, l)$. Thus, $\theta_{i}$ from eq. 2 is the incident angle of the beam on the $(h k l)$ plane, and the Bragg relation follows :

$$
2 d_{h k l} \sin \theta_{i}=\lambda_{0},
$$

where $d_{h k l}$ is the planar spacing of planes $(h k l)$.

This relation is valid for an ideally perfect crystal. Actually, even for very good monocrystals, the reflection is not purely monochromatic and the angular range over which the crystal reflects the beam has a small width. Nevertheless, in practice and for monocrystals, this angular range is very 
small (a few arcseconds) and we will now consider that the measurements integrate over this angular range (and/or over the energy range).

In that case, the integrated power $P$ is given by :

$$
P=I_{0} Q \delta V,
$$

where $I_{0}$ is the beam intensity, $\delta V$ the diffracting volume and $Q$ the diffraction efficiency $[10]$ :

$$
Q=\left(\frac{r_{e}}{V_{c}}\right)^{2} K^{2} \frac{\left|F_{s}(\boldsymbol{\Delta} \mathbf{k})\right|^{2} \lambda_{0}^{3}}{\sin 2 \theta_{i}}
$$

In this formula, the different terms correspond to :

$r_{e}$ : classical electron radius $\left(\approx 2.81794 \cdot 10^{-13} \mathrm{~cm}\right)$.

$V_{c}$ : volume of the elementary cell $\left(=a^{3}\right.$ for a cubic cell of parameter $\left.a\right)$.

$F_{s}$ : structure factor (see below for its calculation).

$\frac{\lambda_{0}^{3}}{\sin 2 \theta_{i}}$ : Lorentz factor, due to the integration of the flux in the diffracted peak.

$K^{2}$ : polarization factor, between $1-\sin ^{2} 2 \theta_{i}$ and 1 (see below).

For compactness, it is useful to define the so-called extinction length (whose meaning will be clarified latter) :

$$
t_{\text {ext }}=\frac{V_{c}}{r_{e}\left|F_{s}\right| \lambda_{0}} \approx 0.28622 \frac{E(\mathrm{keV}) V_{c}\left(\AA^{3}\right)}{\left|F_{s}\right|} \mu \mathrm{m}
$$

One can then write the value of $Q$ in the form :

$$
Q=K^{2} \frac{\lambda_{0}}{t_{e x t}^{2} \sin 2 \theta_{i}}
$$

Laue diffraction and polarization Let us consider a linearly polarized incident beam with a polarization angle $\phi$, defined as the angle between the electromagnetic vector and the "scattering plane" (i.e the plane defined by the incident and reflected beams). The polarization direction of the scattered beam $\left(\phi^{\prime}\right)$ is then given by [11] :

$$
\begin{aligned}
\sin \phi^{\prime} & =\sin \phi / \sqrt{1-\sin ^{2} 2 \theta_{i} \cos ^{2} \phi} \\
& =\sin \phi\left(1+2 \theta_{i}^{2} \cos ^{2} \phi\right)+O\left(\theta_{i}^{4}\right)
\end{aligned}
$$

So, strictly speaking, the polarization angle is unchanged $\left(\phi^{\prime}=\phi\right)$ only for parallel $(\phi=0)$ or normal $(\phi=\pi / 2)$ polarization.

Additionally, the intensity of the scattered beam depends on $\phi$. More precisely, the polarization factor $K^{2}$ in eq. 6 is given by $K^{2}=1-\sin ^{2} 2 \theta_{i} \cos ^{2} \phi$. The polarization modulation is then maximum for $\phi=\pi / 2(K=1)$ and minimum for a parallel polarization. In case of an unpolarized beam (e.g. an X-ray tube), $K^{2}$ should be averaged on the $\phi$ values : $\left\langle K^{2}\right\rangle_{\phi}=1-\sin ^{2} 2 \theta_{i} / 2=$ $\left(1+\cos ^{2} 2 \theta_{i}\right) / 2$. 
For example, at $500 \mathrm{keV}$ and considering a $\mathrm{Cu}_{111}$ plane, the relative polarization rotation and attenuation are of the order of $10^{-4}$. Thus, except for low energy photons $(E<50 \mathrm{keV})$, the Laue lens is practically insensitive to polarization, though its direction is preserved and can be analyzed by the detector (e.g. through the Compton effect).

Consequently, neglecting the polarization effect, $Q$ can be simplified, using the Bragg relation (eq. 4) :

$$
Q \approx\left(\frac{r_{e}}{V_{c}}\right)^{2}\left|F_{s}(\Delta \mathbf{k})\right|^{2} \lambda_{0}^{2} d_{h k l}=\frac{d_{h k l}}{t_{e x t}^{2}}
$$

Finally, the reflectivity is defined as the diffracted integrated power (eq. 5) divided by the incident integrated power $\left(P_{i}=I_{0} \Delta x \Delta y \cos \theta_{i}\right.$, where $\Delta x$ and $\Delta y$ are the dimensions of the crystal perpendicular to the propagation direction). Since $\delta V=\Delta x \Delta y t_{0}$, where $t_{0}$ is the thickness of the crystal, one has :

$$
R_{m}=\frac{P}{P_{i}}=Q \frac{t_{0}}{\cos \theta_{i}}
$$

According to this formula, $Q$ represents the reflectivity per unit of length and $t_{0} / \cos \theta_{i}$ is the effective thickness, as seen by the incident beam.

\subsection{Calculation of the structure factor}

The structure factor $F_{s}(\boldsymbol{\Delta} \mathbf{k})$ is an imaginary coefficient which quantifies the scattering "efficiency" of an elementary cell, i.e considering the diffusion by each atom of the cell. $F_{s}$ is composed of two terms : the scattering factor for a given atom and the geometrical factor, taking into account the interferences between atoms.

The atomic scattering factor The atomic scattering factor $(f)$ represents the amplitude of the wave scattered by a single atom, expressed in unit of the contribution from a single electron. As a general rule $f$ depends on $\sin \theta_{i} / \lambda_{0}$ and approaches the electron number $(\mathrm{Z})$ for small values of this ratio. The exact calculation of the atomic scattering factor for any $\sin \theta_{i} / \lambda_{0}$ is usually difficult since it requires to consider the coherent diffusion by each electron of an atom, taking into account screening factors and quantum physics. Theoretical results exist (e.g the Hartree and Thomas-Fermi methods) but it is much easier (and efficient) to use the following formulas, valid for small incident angles (i.e less than $\approx 10 \mathrm{deg}$, so this condition is always satisfied in the $\mathrm{X}$ and gamma ray regimes) :

$$
f=c+\sum_{i=1}^{4} a_{i} e^{-b_{i}\left(\frac{\sin \theta_{i}}{\lambda_{0}}\right)^{2}},
$$

that is, using the Bragg relation :

$$
f=c+\sum_{i=1}^{4} a_{i} e^{-\frac{b_{i}}{4 d_{h k l}^{2}}}
$$


The $c, a_{i}$ and $b_{i}$ (in $\AA^{2}$ ) coefficients can be found in tables. As an example, table 1 gives, for a few elements, these values, as extracted from [12].

\begin{tabular}{lcccc}
\hline Element & Gold & Copper & Silicon & Germanium \\
\hline$a_{1}$ & 16.8819 & 13.3380 & 5.6627 & 16.0816 \\
$a_{2}$ & 18.5913 & 7.1676 & 3.0716 & 6.3747 \\
$a_{3}$ & 25.5582 & 5.6158 & 2.6245 & 3.7068 \\
$a_{4}$ & 5.8600 & 1.6735 & 1.3932 & 3.6830 \\
$c$ & 12.0658 & 1.1910 & 1.2471 & 2.1313 \\
$b_{1}$ & 0.4611 & 3.5828 & 2.6652 & 2.8509 \\
$b_{2}$ & 8.6216 & 0.2470 & 38.6634 & 0.2516 \\
$b_{3}$ & 1.4826 & 11.3966 & 0.9169 & 11.4468 \\
$b_{4}$ & 36.3956 & 64.8126 & 93.5458 & 54.7625 \\
\hline
\end{tabular}

Table 1 Atomic scattering coefficients

The structure factor The atomic scattering factor gives the intensity of the beam, as diffused by a given atom. Since these atoms are in a crystal lattice, it is necessary to add the waves scattered by all the atoms of an elementary cell. This summation results in the structure factor (since it depends on the geometry of the lattice) :

$$
F_{s}(\boldsymbol{\Delta} \mathbf{k})=\sum_{m=1}^{n} e^{-i \boldsymbol{\Delta} \mathbf{k} \cdot\left(u_{m} \mathbf{a}+v_{m} \mathbf{b}+w_{m} \mathbf{c}\right)} f_{m}
$$

and, using the Laue conditions relation (eq. 3) :

$$
F_{s}=\sum_{m=1}^{n} e^{-2 i \pi\left(h u_{m}+k v_{m}+l w_{m}\right)} f_{m},
$$

where $n$ is the number of atoms per elementary lattice, the $\mathrm{m}^{\text {th }}$ atom being at the position $\left(u_{m}, v_{m}, w_{m}\right)$ in the unit cell defined by the lattice vectors $(\mathbf{a}, \mathbf{b}, \mathbf{c}) . f_{m}$ is the atomic scattering factor of the $\mathrm{m}^{\text {th }}$ atom. When all the atoms of the lattice are of the same kind (e.g copper, germanium, etc), the structure factor can be written as :

$$
\begin{aligned}
F_{s} & =f \sum_{m=1}^{n} e^{-2 i \pi\left(h u_{m}+k v_{m}+l w_{m}\right)} \\
& =f G
\end{aligned}
$$

$G$ is the geometrical factor, only depending on the positions of the atoms in the lattice and on the Miller indexes. The coordinates of the four atoms of a fcc lattice (e.g. copper) are given in table 2 . With these values, the geometrical factor is :

$$
\begin{aligned}
G_{f c c} & =1+\cos \pi(h+k)+\cos \pi(h+l)+\cos \pi(k+l) \\
& = \begin{cases}4 & \text { if } h, k, l \text { all odd or even } \\
0 & \text { otherwise }\end{cases}
\end{aligned}
$$




\begin{tabular}{lccc}
\hline & $\mathrm{u}$ & $\mathrm{v}$ & $\mathrm{w}$ \\
\hline Origin & 0 & 0 & 0 \\
Center face (100) & 0 & 0.5 & 0.5 \\
Center face (010) & 0.5 & 0 & 0.5 \\
Center face (001) & 0.5 & 0.5 & 0 \\
\hline
\end{tabular}

Table 2 Coordinates of the atoms in a face centered cubic lattice

For a diamond lattice (e.g germanium) there are two fcc lattices with an offset of $\left(\frac{1}{4}, \frac{1}{4}, \frac{1}{4}\right)$ :

$$
\begin{aligned}
G_{\text {diam }}= & G_{f c c}\left(1+e^{-i \frac{\pi}{2}(h+k+l)}\right) \\
= & \begin{cases}4\left(1+e^{-i \frac{\pi}{2}}\right) & \text { if } h, k, l \text { odd and } h+k+l=1[4] \\
4\left(1-e^{-i \frac{\pi}{2}}\right) & \text { if } h, k, l \text { odd and } h+k+l=3[4] \\
8 & \text { if } h, k, l \text { even et } h+k+l=0[4] \\
0 & \text { otherwise }\end{cases}
\end{aligned}
$$

This geometrical factor shows that, due to destructive interferences, some crystal planes cannot reflect the beam.

3.2 Effect of thermal displacements on diffraction efficiency : the Debye factor

Because of the thermal movements of the atoms in a solid, the effective emitted wave should be averaged over all the vibrational displacements. Compared to the ideal case of perfectly fixed atoms, the structure factor should then be corrected :

$$
F_{s}=\sum_{m=1}^{n} e^{-2 i \pi\left(h u_{m}+k v_{m}+l w_{m}\right)} f_{m} e^{-M_{m}},
$$

where $M_{m}$ is related to the mean amplitude of the atomic displacements $\left(\Delta X_{m}\right)[7,9]$ :

$$
\begin{aligned}
M_{m} & =\frac{8 \pi^{2} \sin ^{2} \theta_{i} \Delta X_{m}^{2}}{3 \lambda_{0}^{2}}=B_{m}\left(\frac{\sin \theta_{i}}{\lambda_{0}}\right)^{2}, \\
& =\frac{B_{m}}{4 d_{h k l}^{2}} \quad \text { using the Bragg relation }
\end{aligned}
$$

Eq. 20 shows that the smaller the planar spacing $\left(d_{h k l}\right)$, the bigger $M_{m}$ and the smaller the intensity of the diffracted beam. Physically, this means that the thermal displacement "blurs" the effective distance between the planes, this effect being proportionally more important when $d_{h k l}$ is small.

$\Delta X_{m}$ may be different for different kinds of atoms and $(h k l)$ planes. If there is only one kind of atom, the diffracted intensity should be multiplied by the Debye (or Debye-Waller) factor $D=e^{-2 M}$. 
Moreover, in the case of a cubic crystal containing only one kind of atom (e.g copper, germanium), $\Delta X$ can be calculated using $[7,9]$ :

$$
\begin{aligned}
& \Delta X^{2}=\frac{9 h^{2} \mathcal{N}_{a}}{4 \pi^{2} M_{a} k \Theta}\left[\frac{1}{4}+\frac{T}{\Theta} \varphi\left(\frac{\Theta}{T}\right)\right] \\
& \text { where } \varphi\left(\frac{\Theta}{T}\right)=\frac{T}{\Theta} \int_{0}^{\frac{\Theta}{T}} \frac{y}{e^{y}-1} d y
\end{aligned}
$$

In this equation :

$h$ is the Planck constant

$k$ is the Boltzman constant

$\mathcal{N}_{a}$ is the Avogadro number

$M_{a}$ is the atomic mass

$T$ is the temperature of the crystal

$\Theta$ is a characteristic temperature.

Numerically, it follows :

$$
B=\frac{1.149 \cdot 10^{4} \mathrm{~g} \cdot \mathrm{mol}^{-1} \cdot \mathrm{K}}{M_{a} \Theta}\left[\frac{1}{4}+\frac{T}{\Theta} \varphi\left(\frac{\Theta}{T}\right)\right] \quad \AA^{2}
$$

Additionally, $\frac{1}{4}+\frac{T}{\Theta} \varphi\left(\frac{\Theta}{T}\right)$ can be roughly approximated, with an absolute error smaller than 0.025 , by :

$$
\frac{1}{4}+\frac{T}{\Theta} \varphi\left(\frac{\Theta}{T}\right) \approx \frac{T}{\Theta}+\frac{1}{4} e^{-4 \frac{T}{\Theta}}
$$

$\Theta$ is not quite the same as the Debye temperature from the specific heat theory. Nevertheless, the difference is small and to a first approximation the tabulated Debye temperature can be used.

\section{Limitations and corrections to the kinematical model}

At this point, all the elements are defined to calculate $Q$ (in eq. 10) in the ideal case of the kinematical theory which is based on a few assumptions :

1. The refraction index of the crystal is very close to the refraction index of the environment. This assumption is always valid for $\mathbf{X}$ and $\gamma$ rays.

2. The absorption in a single crystal block is negligible, each atom receiving the same intensity from the source. This assumption is valid if the thickness of the crystal block is small compared to the mean free path of the material.

3. The interferences between direct and diffracted beams have been neglected in the crystal. This assumption, not always valid and difficult to estimate, is discussed below. 
4.1 The extinction effect

The direct and diffracted beams interfere alternatively constructively and destructively, due to their phase difference, when propagating through the crystal. After a given thickness, the two effects are in equilibrium and increasing the size of the crystal does not result in a higher diffracted intensity (i.e an increase of $R_{m}$, as given in eq. 10) : this effect is called the extinction effect. For a thin crystal, the above equations (from the kinematical theory) are valid. For a thick crystal, one should use the dynamical theory which gives for the integrated reflectivity in Laue geometry [10] :

$$
R_{d y n}=\frac{r_{e}}{V_{c}} \frac{1+\left|\cos 2 \theta_{i}\right|}{2} \frac{\sqrt{D}\left|F_{s}\right| \lambda_{0}^{2}}{2 \sin 2 \theta_{i}} \frac{t_{0}}{\cos \theta_{i}}
$$

In practice, one has :

$$
R_{k i n}>R_{m}>R_{d y n}
$$

where $R_{k i n}$ is the integrated reflectivity from the kinematical theory, given by eq. 10 , and $R_{m}$ is the measured integrated reflectivity. One defines the parameter $A=\frac{K}{\cos \theta_{i}} \frac{t_{0}}{t_{e x t}}$, where $K$ is the polarization factor : $K=\frac{1+\left|\cos 2 \theta_{i}\right|}{2}$ and $K^{2}=\frac{1+\cos ^{2} 2 \theta_{i}}{2}$ for an unpolarized beam, and $t_{0}$ is the thickness of the crystal block. With these definitions, the integrated reflectivity in Laue geometry can be written as [10] :

$$
R_{m}=\frac{K}{2 \sin 2 \theta_{i}} \frac{\lambda_{0}}{t_{\text {ext }}} \int_{0}^{2 A} J_{0}(\rho) d \rho
$$

In this formula, $J_{0}$ is the 0 -order Bessel function. Figure 2 shows the variations of $I_{0}(2 A)=\int_{0}^{2 A} J_{0}(\rho) d \rho$ as a function of $A$. This curve increases until

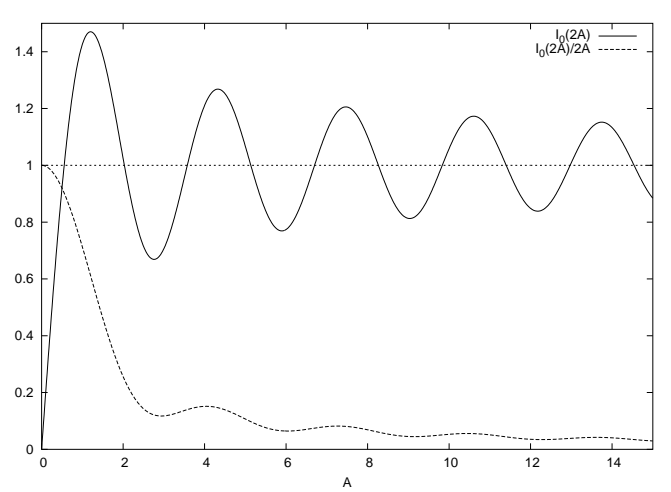

Fig. 2 Relative variations of the integrated reflectivity with crystal thickness.

a maximum for $A \approx 1.202$ is reached. It then oscillates around the value of 
1 , showing the saturation of the reflectivity (Pendellösung effect). In the two extreme cases, one has:

$$
I_{0}(2 A)= \begin{cases}2 A & \text { if } A \ll 1 \\ 1 & \text { if } A \gg 1\end{cases}
$$

The transition from the kinematical to the dynamical theory is then driven by the extinction length (eq. 7 ). When $t_{0} \ll t_{e x t}$ the crystal is thin and the kinematical theory applies. When $t_{0} \gg t_{\text {ext }}$ the crystal is thick and one should use the dynamical theory. Numerically one can use the following approximation for $I_{0}(x)$ :

$$
\begin{aligned}
I_{0}(x)= & \begin{cases}\frac{x N\left(x^{2}\right)}{D\left(x^{2}\right)} & \text { if } x \leq 8 \\
1.0+\sqrt{\frac{2}{\pi x}}\left(P\left(\left(\frac{8}{x}\right)^{2}\right) \sin \left(x-\frac{\pi}{4}\right)-\frac{8}{x} Q\left(\left(\frac{8}{x}\right)^{2}\right) \cos \left(x-\frac{\pi}{4}\right)\right) & \text { if } x>8\end{cases} \\
N(x)= & 144725+15706.9297 x-73.034996 x^{2}-33.49237 x^{3} \\
& +1.30278 x^{4}-0.0081205 x^{5} \\
D(x)= & 144725+27781.8340 x+1779.3622 x^{2}+39.6246 x^{3} \\
& +0.323458 x^{4}+0.0087508 x^{5} \\
P(x)= & 1.0-0.015928 x+0.00334056 x^{2}-0.00309143 x^{3}+0.00172450 x^{4} \\
Q(x)= & 0.0781342-0.0050871 x+0.0010301 x^{2}-0.0000876611 x^{3} \\
& +0.0000806711 x^{4}
\end{aligned}
$$

Finally, in the intermediate regime and considering an unpolarized beam, equation 10 for the integrated reflectivity is always valid by replacing $Q$ with $Q^{\prime}$ defined as follows (in Laue geometry) [10] :

$$
\begin{aligned}
Q^{\prime} & =\frac{I_{0}\left(2 A_{0}\right)+\left|\cos 2 \theta_{i}\right| I_{0}\left(2 A_{0}\left|\cos 2 \theta_{i}\right|\right)}{2 A_{0}\left(1+\cos ^{2} 2 \theta_{i}\right)} Q \\
A_{0} & =\frac{A}{K}=\frac{t_{0}}{\cos \theta_{i} t_{\text {ext }}}
\end{aligned}
$$

Given the small incident angles of $\mathrm{X}$ and $\gamma$ rays :

$$
\begin{aligned}
Q^{\prime} & \approx \frac{I_{0}\left(2 A_{0}\right)}{2 A_{0}} Q \approx \frac{I_{0}\left(2 A_{0}\right)}{2 A_{0}} \frac{d_{h k l}}{t_{e x t}^{2}} \\
& \approx 3.4939 \frac{I_{0}\left(2 A_{0}\right)}{2 t_{0}} \frac{d_{h k l}(\AA)}{\left(E(\mathrm{keV}) V_{c}\left(\AA^{3}\right)\right)}\left|F_{s}\right| \mathrm{cm}^{-1}
\end{aligned}
$$

These equations clearly show that the integrated reflectivity per unit of length decreases when the blocks' thickness increases. Since the "mosaic" crystals are a conglomerate of such crystallites, one should keep their size $t_{0}$ as small as possible. Unfortunately, the thickness of the crystallites depends on the growing process and this parameter appears difficult to control. 
The extinction length is therefore the transition thickness from "thin" to "thick" crystals. For a given material and diffraction plane, the extinction length is proportional to the energy. Thus, a crystal which is "thick" at low energy may become "thin" (and so better) at higher energy. The table 3 gives the extinction length for some materials and diffracting planes at $500 \mathrm{keV}$ and $293 \mathrm{~K}$ (Debye factor). A simple proportional factor applies for any other energy.

\begin{tabular}{lcccccccc}
\hline & 111 & 200 & 220 & 222 & 311 & 331 & 333 & 400 \\
\hline Copper & 78.82 & 85 & 109.1 & 133.8 & 127.6 & 178.5 & 230.6 & 159.1 \\
Silicon & 395.6 & & 342.6 & & 521.8 & 612.1 & 708.2 & 408.8 \\
Germanium & 169.1 & & 139.7 & & 212.1 & 249.7 & 288.5 & 166.8 \\
Gold & 37.6 & 39.5 & 46.4 & 52.8 & 51.2 & 63.5 & 75.7 & 58.9 \\
\hline
\end{tabular}

Table 3 Extinction length in $\mu \mathrm{m}$ at $293 \mathrm{~K}$ and $500 \mathrm{keV}$ for various elements

\section{Macroscopic crystals : the Darwin model}

According to the previous section, the integrated power of a "perfect" crystal saturates after a few tenth of $\mathrm{mm}$, due to the extinction effect. Nevertheless, the measurements performed on "real" macroscopic crystals (of thickness $T_{0}$ ) can be explained neither by the kinematical nor by the dynamical model of a "perfect" crystal. Actually, the angular (or energy) acceptance can be relatively large (up to a few degrees), and the integrated reflectivity is much higher than expected from a perfect crystal with a thickness $T_{0} \gg t_{\text {ext }}$. Darwin (see $[13,14,15,10]$ ) proposed that a macroscopic crystal is actually an agglomerate of small, perfect crystals. The angular orientation of these small crystals is randomly distributed (see fig. 3 ). Thus, the diffracted beams

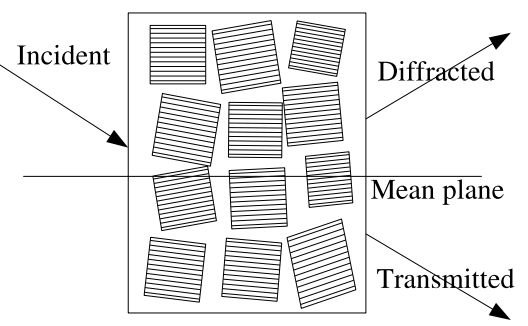

Fig. 3 Scheme of a mosaic crystal

from the individual crystals blocks do not interfere and the extinction effect (mostly) cancels out. This mosaic model is defined through the angular distribution of the crystallites $W(\boldsymbol{\Delta})$ (where $\boldsymbol{\Delta}$ is the orientation of the block) and their mean thickness $\left(t_{0}\right)$. Usually, the angular spread is projected on a 
given diffraction plane, and is assumed to be normally distributed :

$$
W(\Delta)=\frac{1}{\sqrt{2 \pi} \eta} e^{-\frac{\Delta^{2}}{2 \eta^{2}}}
$$

The mosaicity is then the FWHM of this distribution : $m=2 \sqrt{2 \ln 2} \eta$.

Another assumption of the mosaic model is that the mosaicity is much bigger than the angular reflectivity range of a single crystal. This assumption is usually valid for mosaicities above $\approx 10^{\prime \prime}$. In this case, the reflectivity power of a layer of thickness $t_{0}$ is given by $\mathcal{R} \approx W\left(\theta-\theta_{B}\right) R_{m}$ where $\theta$ is the incident angle of the beam, $\theta_{B}$ is the Bragg angle corresponding to the beam energy and the mean orientation of the diffraction planes, $R_{m}$ is from eq. 10 .

$\sigma$ is then defined as the integrated reflectivity per unit of length : $\sigma=$ $W\left(\theta-\theta_{b}\right) R_{m} / t_{0}$.

In case of crystallites of negligible size (i.e. smaller than the extinction length, $\left.t_{0} \ll t_{\text {ext }}\right)$, the crystal is "ideally imperfect", corresponding to the optimum reflectivity that can be obtained according to the Darwin model. Otherwise, the extinction effect (see $\S 4.1$ ) should be taken into account. So, using the appropriate expression for $Q$, depending on the mean thickness $t_{0}$ (eq. 6 or 30 ), one obtains :

$$
\cos \theta_{B} \sigma \approx \sigma \approx \begin{cases}W\left(\theta_{B}-\theta\right) Q & \text { if } t_{0} \ll t_{\text {ext }} \\ W\left(\theta_{B}-\theta\right) Q^{\prime} & \text { otherwise }\end{cases}
$$

When propagating through the crystal, the beam can be absorbed (linear absorption $\mu$ ) or diffracted $(\sigma)$. Additionally, assuming the crystal to be homogeneous, the diffraction probability is the same from the incident to the diffracted direction, or vice versa. So, defining $\mathcal{P}_{0}(T)$, resp. $\mathcal{P}(T)$, as the power (or intensity) of the beam in the incident, resp. diffracted, direction at the depth $T$, the following set of differential equations follows (in Laue geometry) [10] :

$$
\begin{aligned}
d \mathcal{P}_{0} & =-\left(\frac{\mu}{\cos \theta}+\sigma\right) \mathcal{P}_{0} d T+\sigma \mathcal{P} d T \\
d \mathcal{P} & =-\left(\frac{\mu}{\cos \theta}+\sigma\right) \mathcal{P} d T+\sigma \mathcal{P}_{0} d T \\
\mathcal{P}_{0}(T=0) & =\mathcal{P}_{0}(0), \mathcal{P}(T=0)=0
\end{aligned}
$$

which leads to :

$$
\begin{aligned}
& \frac{\mathcal{P}\left(T_{0}\right)}{\mathcal{P}_{0}(0)}=\sinh \left(\sigma T_{0}\right) e^{-\left(\frac{\mu}{\cos \theta}+\sigma\right) T_{0}}=\frac{1}{2}\left(1-e^{-2 \sigma T_{0}}\right) e^{-\mu \frac{T_{0}}{\cos \theta}} \\
& \frac{\mathcal{P}_{0}\left(T_{0}\right)}{\mathcal{P}_{0}(0)}=\cosh \left(\sigma T_{0}\right) e^{-\left(\frac{\mu}{\cos \theta}+\sigma\right) T_{0}}=\frac{1}{2}\left(1+e^{-2 \sigma T_{0}}\right) e^{-\mu \frac{T_{0}}{\cos \theta}}
\end{aligned}
$$

Usually, the Bragg angle is small and so :

$$
\begin{aligned}
\frac{\mathcal{P}\left(T_{0}\right)}{\mathcal{P}_{0}(0)} & \approx \sinh \left(\sigma T_{0}\right) e^{-(\mu+\sigma) T_{0}} \\
& \approx \frac{1}{2}\left(1-e^{-2 \sigma T_{0}}\right) e^{-\mu T_{0}}
\end{aligned}
$$


Formula 37, along with equation 33, is of constant use to estimate the diffraction efficiency of a mosaic crystal, and by extension, the efficiency of a Laue lens.

There are usually two kinds of experiments performed in the laboratory to measure the reflectivity of a mosaic crystal :

- The incident beam is monochromatic $\left(\theta_{B}\right.$ is constant) and we consider the variation of the reflectivity while changing the orientation (angle $\theta$ ) of the crystal. This method, called a rocking curve, is adequate to precisely measure the reflectivity function, since the variation in $\theta$ doesn't influence any other parameter.

- The position of the crystal is fixed ( $\theta$ is constant), and the variation of reflectivity with the energy of the beam (variation of $\theta_{B}$ ) is measured. In this case, the diffracted spectrum peaks at the energy $E_{0}$ that satisfies the Bragg relation for the mean plane orientation $\left(\lambda_{0}=2 d_{h k l} \sin \theta_{0}\right)$. Besides, there are also $2^{\text {nd }}$ order variations on $\mu$ and $Q$. These variations can be neglected if the diffracted energy bandwidth is small compared to the mean energy (i.e $\Delta E \ll E_{0}$ ) and the two experiments are then almost equivalent.

\subsection{Some properties of the diffraction curve}

In this section, the orientation of the crystallites is assumed to be normally distributed :

$$
W(\Delta \theta)=2 \sqrt{\frac{\ln (2)}{\pi}} \frac{1}{m} e^{-\ln (2)\left(\frac{\Delta \theta}{m / 2}\right)^{2}},
$$

Then, considering equation 37 , it is useful to define the following dimensionless parameters :

$$
\alpha=4 \sqrt{\frac{\ln (2)}{\pi}} \frac{d_{h k l} T_{0}}{t_{e x t}^{2} m}, u=\frac{\Delta \theta}{m / 2}
$$

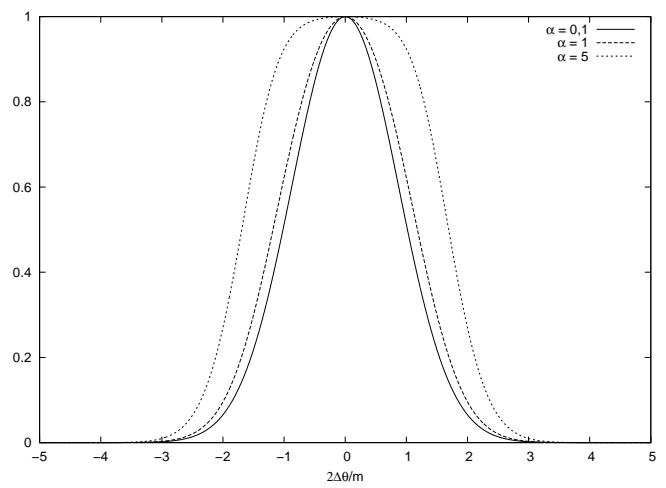

Fig. 4 Normalized reflectivity curves for different values of $\alpha$. 
With these reduced parameters, the reflectivity curve has the form (see also fig. 4) :

$$
\frac{\mathcal{P}\left(T_{0}\right)}{\mathcal{P}_{0}(0)}=\frac{1}{2}\left(1-e^{-\alpha e^{-\ln (2) u^{2}}}\right) e^{-\mu T_{0}},
$$

As previously mentioned, in the case of measurements with a constant incident angle but a variable wavelength, the variation of $Q$ and $\mu$ in the diffracted energy bandwidth can usually be neglected. With this assumption, the FWHM and maximum of the diffraction curve are given by :

$$
\begin{aligned}
\Delta u_{F W H M} & =2 \sqrt{\frac{-\ln \left(-\frac{1}{\alpha} \ln \left(\frac{1}{2}\left(1+e^{-\alpha}\right)\right)\right)}{\ln (2)}} \\
\left(\frac{\mathcal{P}\left(T_{0}\right)}{\mathcal{P}_{0}(0)}\right)_{\max } & =\left(\frac{\mathcal{P}\left(T_{0}\right)}{\mathcal{P}_{0}(0)}\right)_{u=0}=\frac{1}{2}\left(1-e^{-\alpha}\right) e^{-\mu T_{0}}
\end{aligned}
$$

From these equations, one can demonstrate that $\Delta u_{F W H M}>2$ (i.e $\Delta \theta_{F W H M}>$ $m$ ) whatever the value of $\alpha$. In other words, the FWHM of the rocking curve is always greater than the mosaicity. Additionally, it tends to the normal angular distribution of the crystallites for $\alpha \rightarrow 0$. More precisely, the Taylor series expansion around $\alpha=0$ of the diffraction curve and its FWHM gives :

$$
\begin{gathered}
\frac{\mathcal{P}\left(T_{0}\right)}{\mathcal{P}_{0}(0)}=\frac{1}{2} \sum_{k=1}^{+\infty}(-1)^{k-1} \frac{e^{-k \ln (2) u^{2}}}{k !} \alpha^{k} \\
\Delta u_{F W H M} \approx 2\left(1+\frac{\alpha}{8 \ln (2)}\left(1+\left(1-\frac{1}{16 \ln (2)}\right) \frac{\alpha}{8 \ln (2)}\right)\right)
\end{gathered}
$$

It is also often desirable to calculate the total flux diffracted by a crystal, i.e the integration of $\frac{\mathcal{P}\left(T_{0}\right)}{\mathcal{P}_{0}(0)}$ over $\theta$ (or $\lambda$ ). Unfortunately, this integration cannot be done analytically, but an good approximation can be obtained by multiplying the FWHM of the diffraction curve with its maximum amplitude. Mathematically, this means :

$$
\begin{aligned}
\mathcal{R}^{u}(\alpha) & =\int_{-\infty}^{+\infty} \frac{1}{2}\left(1-e^{-\alpha e^{-\ln (2) u^{2}}}\right) d u \\
& \approx\left(1-e^{-\alpha}\right) \sqrt{\frac{-\ln \left(-\frac{1}{\alpha} \ln \left(\frac{1}{2}\left(1+e^{-\alpha}\right)\right)\right)}{\ln (2)}} \\
\mathcal{R}^{\theta} & =\int_{-\infty}^{+\infty} \frac{\mathcal{P}\left(T_{0}\right)}{\mathcal{P}_{0}(0)} d \Delta \theta \\
& \approx \frac{m}{2} e^{-\mu T_{0}} \int_{-\infty}^{+\infty} \frac{1}{2}\left(1-e^{\left.-\alpha e^{-\ln (2) u^{2}}\right) d u}\right. \\
& \approx \frac{m}{2} \mathcal{R}^{u}(\alpha) e^{-\mu T_{0}}=2 \sqrt{\frac{\ln (2)}{\pi}} Q T_{0} \frac{\mathcal{R}^{u}(\alpha)}{\alpha} e^{-\mu T_{0}} \\
\mathcal{R}^{\lambda} & =\int_{-\infty}^{+\infty} \frac{\mathcal{P}\left(T_{0}\right)}{\mathcal{P}_{0}(0)} d \lambda \approx 2 d_{h k l} \mathcal{R}^{\theta} \\
& \approx d_{h k l} m \mathcal{R}^{u}(\alpha) e^{-\mu T_{0}}=4 \sqrt{\frac{\ln (2)}{\pi}} Q T_{0} d_{h k l} \frac{\mathcal{R}^{u}(\alpha)}{\alpha} e^{-\mu T_{0}},
\end{aligned}
$$


where the upper indexes indicate the integration parameter $(\theta, \lambda$ or $u) \cdot \mathcal{R}^{u}$ is dimensionless but this is not the case of $\mathcal{R}^{\theta}$ and $\mathcal{R}^{\lambda}$ which are, respectively, the integrated flux considering a rocking curve or a continuum energy emission, and are consequently expressed in photons $(/ \mathrm{s})$. So, $m$ should be expressed in arcseconds in $\mathcal{R}^{\theta}$ if the incident flux is in photons/arcsec (we are only interested in the projection of the incident flux in the meridian plane). In $\mathcal{R}^{\lambda}, m$ should be expressed in radians (since it indirectly comes from the linearization of the Bragg relation) and $d_{h k l}$ in $\AA$ if the incident, polychromatic flux is in (or converted to, using the $E_{0}^{2} / h c$ factor) photons $/ \AA$.

The validity of this approximation can be checked on fig. 5 , where the numerical and approximated values of $\mathcal{R}^{u}(\alpha)$ and $\mathcal{R}^{u}(\alpha) / \alpha$ are plotted. This approximation is also used and compared to a $\mathrm{MC}$ simulation of a broad band Laue lens in a accompanying article ([3], this volume).

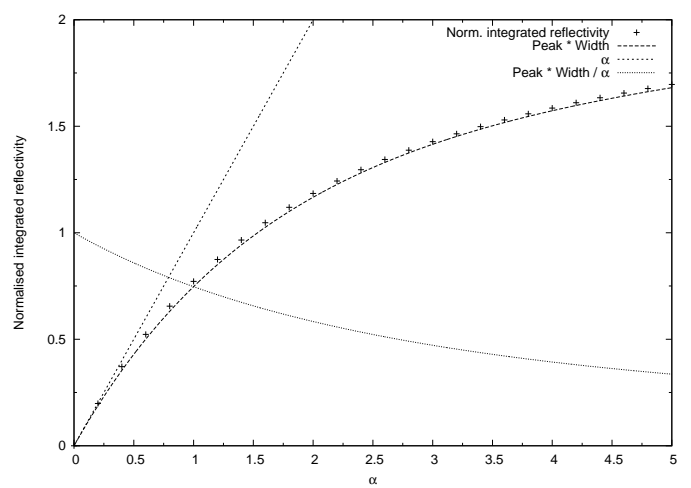

Fig. 5 Normalized integrated reflectivity (without absorption) of a mosaic crystal. Crosses represent the numerical integration, the dot line is the approximated analytical model

Alternatively, integrating the Taylor series of the diffraction curve (see eq. 42) gives the expansion of $\int_{0}^{u} \frac{1}{2}\left(1-e^{-\alpha e^{-\ln (2) v^{2}}}\right) d v$ :

$$
\begin{aligned}
F_{\alpha}(u) & =\int_{0}^{u} \frac{1}{2}\left(1-e^{-\alpha e^{-\ln (2) v^{2}}}\right) d v \\
& =\frac{1}{4} \sqrt{\frac{\pi}{\ln (2)}} \sum_{k=1}^{+\infty}(-1)^{k-1} \frac{\operatorname{erf}(\sqrt{k \ln (2)} u)}{\sqrt{k} k !} \alpha^{k}
\end{aligned}
$$

From this equation, the Taylor series expansion of the integrated reflectivity $\left(\mathcal{R}^{u}(\alpha)=2 F_{\alpha}(\infty)\right)$ follows :

$$
\mathcal{R}^{u}(\alpha)=\frac{1}{2} \sqrt{\frac{\pi}{\ln (2)}} \sum_{k=1}^{+\infty} \frac{(-1)^{k-1}}{\sqrt{k} k !} \alpha^{k}
$$


The above equations (see also fig. 5) demonstrate that $\mathcal{R}^{u}(\alpha) / \alpha$ decreases with $\alpha$ and $\mathcal{R}^{u}(\alpha) / \alpha \approx 1$ if $\alpha \ll 1$. This means that the integrated reflectivity, for a given thickness $T_{0}$, asymptotically increases with the mosaicity, approaching a constant value if $m \gg \frac{2 d_{h k l} T_{0}}{t_{e x t}^{2}}$. This condition is not always satisfied though. Especially, the parameters optimization can often lead to values for which $\frac{T_{0}}{m} \approx \frac{t_{e x t}^{2}}{d_{h k l}}$.

\section{Crystal optimization strategies}

To get an idea of the "best" parameters of a mosaic crystal, let now try to "optimize" the reflectivity of a single crystal. In the context of the Darwin model, two parameters can eventually be optimized to non trivial values : the mosaicity and the thickness. The size of the crystallites $\left(t_{0}\right)$ should be much smaller than the extinction length (see $\S 4.1$ ), so that the extinction effect is negligible. This latter condition is assumed to be satisfied in the following sections.

6.1 Maximum peak reflectivity for a given mosaicity

The mosaicity of a crystal is often set by "external" constraints (e.g the growing process or the energy resolution of the detector in case of a line observation). In that case, optimizing the peak reflectivity (see eq. 41) leads to the following thickness :

$$
T_{m a x}^{\text {peak }}=\frac{\ln \left(1+4 \sqrt{\frac{\ln (2)}{\pi}} \frac{d_{h k l}}{m t_{e x t}^{2} \mu}\right)}{4 \sqrt{\frac{\ln (2)}{\pi}} \frac{d_{h k l}}{m t_{e x t}^{2}}}
$$

Since $\ln (1+x) / x$ is always between 0 and 1 for $x>0$, the optimal thickness is always smaller than the mean free path $1 / \mu$, whatever the mosaicity $m$. Moreover, $T_{\text {max }}^{p e a k}$ increases with $m$ and tends to $1 / \mu$ if $m \gg \frac{2 d_{h k l}}{t_{e x t}^{2} \mu}$.

Since the width of the diffracted peak increases with the crystal thickness, the optimal significance (taking into account the background noise in the diffracted bandwidth) could lead to a slightly different value of $T_{0}$ (see below), smaller than $T_{\max }^{\text {peak }}$.

\subsection{Detection optimization for a broad band emission}

Here, we assume that both mosaicity and crystal thickness are free parameters. The optimization criterion is the detection significance in the energy bandwidth of the crystal. According to the approximation of eq. 44, the signal is proportional to :

$$
P_{S} \propto m f(\alpha) \sqrt{g(\alpha)} e^{-\mu T},
$$


where :

$$
\begin{aligned}
& f(\alpha)=\frac{1}{2}\left(1-e^{-\alpha}\right) \\
& g(\alpha)=-\ln \left(-\frac{\ln \left(\frac{1}{2}\left(1+e^{-\alpha}\right)\right)}{\alpha}\right)
\end{aligned}
$$

Besides, the background level can be estimated by integrating its flux over the diffracted FWHM. Thus, the detection significance (background dominated and assuming Poisson statistics) is proportional to :

$$
n_{\sigma} \propto f(\alpha) \sqrt{m \sqrt{g(\alpha)}} e^{-\mu T}
$$

The maximization of $n_{\sigma}$, w.r.t the mosaicity $m$ and to the thickness $T_{0}$ can be performed analytically and leads to :

$$
\begin{aligned}
T_{\max }^{\sigma} & =\frac{1}{2 \mu} \\
m_{\max }^{\sigma} & =0.57414 \frac{d_{h k l}}{\mu t_{\text {ext }}^{2}} \propto \frac{1}{E^{2}}
\end{aligned}
$$

In that case, it is worth noticing that the optimal thickness only depends on the material and diffracted energy, being half the mean free path, but not on the diffracting plane. Moreover, using this optimal mosaicity as an input for the peak reflectivity maximization procedure of the precedent section, gives $T_{\max }^{\text {peak }} \approx 1.18485 . T_{\max }^{\sigma}$, which confirms that the significance optimization leads to thinner crystals than the peak efficiency optimization.

The optimization criterion is nevertheless quite flat around the optimum (in both cases) and, consequently, even significant departure from optimal parameters (especially concerning the thickness) only slightly affects the "quality" of the crystal.

In practice, other constraints affect the optimal parameters. First, experiments showed that the mean crystallites' size is correlated with the mosaicity : the mosaicity is due to the dislocation density inside the crystal. Hence, the smaller the mosaicity, the bigger the mean thickness of the perfect crystal blocks. Second, the flatness of the optimum may lead to choose thinner crystal in order to save mass budget and/or increase the number of tiles.

\section{Diffraction by an extended crystal}

Up to now, the radial extension of the crystal has been neglected. This is obviously not always valid in practice. Actually, if the source is at finite distance from the crystal, the incident angle, and hence the diffracted energy, depends on the impact radius. Since the mosaicity represents the angular width over which a crystal "line" reflects a monochromatic beam, the radial extension of the crystal is negligible only if the angular size of the crystal (as seen from the source) is small compared to the mosaicity. Given a radial crystal size of $1 \mathrm{~cm}$ and a mosaicity of $1 \mathrm{arcmin}$, this means a source distance 
greater than $34 \mathrm{~m}$ ! This condition may be hard to achieve in laboratory experiments, unless a small slit is mounted in front of the crystal, which may then reduce too much the diffracted flux.

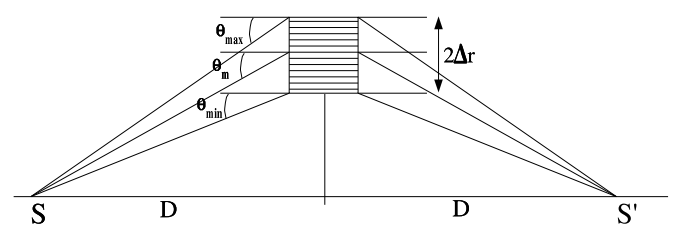

Fig. 6 Diffraction of an extended crystal and a source at finite distance

Nevertheless, some crystal properties can be derived from experiments with an extended crystal and a source at finite distance. Consider the experimental setup represented in fig. 6 : a crystal with a radial extension of $2 \Delta r$ is mounted at a distance $D$ of a source $S$. The detector is placed at the symmetrical point $S^{\prime}$, or at any other place so that the diffracted flux is totally intercepted (but not the direct beam). Moreover the source is assumed to be isotropic, with an intensity $I_{0}$. Two different cases are discussed hereafter : a monochromatic or a continuum source. Besides, the angular distribution of the crystallites is assumed to be Gaussian.

\subsection{Monochromatic source}

In that case, the incoming intensity, projected on the meridian plane is given by $I_{0}$ in units of $\mathrm{ph} \cdot \mathrm{s}^{-1} \cdot \mathrm{cm}^{-1}$. $I_{0}$ is non-zero only for a given wavelength $\left(\lambda_{0}\right)$. If $\theta_{m}$ is the incident angle on the middle of the crystal, then the incident angle at the radius $r$ (varying from $-\Delta r$ to $\Delta r$ ) is given by : $\theta=\theta_{m}+\Delta \theta$, where $\Delta \theta \approx r / D$. Following the approach given in section 5.1, let us define the reduced parameters :

$$
u_{m}=\frac{\theta_{B}-\theta_{m}}{m / 2}, u_{r}=\frac{\Delta \theta}{m / 2}=\frac{r}{D m / 2}, \Delta u_{r}=\frac{\Delta r}{D m / 2},
$$

where $\theta_{B}$ is the Bragg angle for the incident energy $\left(\lambda_{0}\right)$. With these notations and using the definition of $F_{\alpha}$ from eq. 45 , the diffracted intensity is given by :

$$
\begin{aligned}
I_{d i f f} & =\frac{D m}{2} \int_{-\Delta u_{r}}^{+\Delta u_{r}} \frac{1}{2}\left(1-e^{-\alpha e^{-\ln (2)\left(u_{r}-u_{m}\right)^{2}}}\right) d u_{r} e^{-\mu T_{0}} I_{0} \\
& =\frac{D m}{2}\left(F_{\alpha}\left(\Delta u_{r}-u_{m}\right)+F_{\alpha}\left(\Delta u_{r}+u_{m}\right)\right) e^{-\mu T_{0}} I_{0}
\end{aligned}
$$

If the source is close enough and the crystal correctly oriented, then the crystal's mosaicity is much smaller than its angular size and the reflectivity 
curve can be integrated over the full angular range. Mathematically, these conditions are :

$$
\left\{\begin{aligned}
\Delta u_{r}-u_{m} \gg 1 & \Leftrightarrow \frac{\Delta r}{D}-\left(\theta_{B}-\theta_{m}\right) \gg \frac{m}{2} \\
\Delta u_{r}+u_{m} \gg 1 & \Leftrightarrow \frac{\Delta r}{D}+\left(\theta_{B}-\theta_{m}\right) \gg \frac{m}{2}
\end{aligned}\right.
$$

In that case, the diffracted intensity (in $\mathrm{ph} \cdot \mathrm{s}^{-1}$ ) is given by $I_{d i f f}=D \mathcal{R}^{\theta} I_{0}$, where $\mathcal{R}^{\theta}$ is from eq. 44 . The diffraction efficiency is then :

$$
\epsilon_{\text {Mono }}=\frac{I_{d i f f}}{I_{0} 2 \Delta r}=\frac{D}{2 \Delta r} \mathcal{R}^{\theta}
$$

As expected, the diffraction efficiency increases with the source distance (the diffraction "volume" increases).

\subsection{Polychromatic source}

In that case, the incoming, polychromatic intensity, projected on the meridian plane is given by $I_{0}$ in units of $\mathrm{ph} \cdot \mathrm{s}^{-1} \cdot \mathrm{cm}^{-1} \cdot \AA^{-1}$. As usual, the energy bandwidth is assumed to be small so that the crystal properties are almost constant. With this assumption, eq. 52 is still valid for a given wavelength, with $I_{0}$ and $u_{m}$ depending on $\lambda$. Moreover if $I_{0}$ is constant over the energy bandwidth, then integrating over $\lambda$ leads to the total diffracted intensity (in $\left.\mathrm{ph} \cdot \mathrm{s}^{-1}\right)$ :

$$
\begin{aligned}
I_{\text {diff }} & =2 \Delta r \mathcal{R}^{\lambda} I_{0}=4 \Delta r d_{h k l} \mathcal{R}^{\theta} I_{0} \\
& =(2 \Delta r)^{2} \frac{2 d_{h k l}}{D} \epsilon_{\text {Mono }} I_{0},
\end{aligned}
$$

where $\mathcal{R}^{\lambda}$ and $\mathcal{R}^{\theta}$ are from eq. 44 . The total diffracted intensity from a continuum emission is then independent of the source distance. One should nevertheless keep in mind that the energy bandwidth increases as the source comes closer to the crystal.

Additionally and contrary to the monochromatic case, there is no obvious definition of a global efficiency in the polychromatic case, since the diffraction process transforms a continuum emission into a band-limited spectrum. Nevertheless, the mosaic energy spread (that is not due to the crystal angular size) is, according to the Bragg relation, $\Delta \lambda \approx 2 d_{h k l} m$. So, defining the averaged efficiency as the diffracted intensity divided by the incident flux in the mosaic bandwidth, one gets :

$$
\epsilon_{\text {Poly }}=\frac{I_{\text {diff }}}{I_{0} 2 \Delta r 2 d_{h k l} m}=\frac{\mathcal{R}^{\theta}}{m}=\frac{2 \Delta r}{D m} \epsilon_{\text {Mono }}
$$

The conversion factor $(2 \Delta r / D m)$ corresponds to the angular size of the crystal in units of mosaicity (as expected...). From the conditions expressed in eq. 53, it follows that the efficiency for a monochromatic beam is much lower 
than the "efficiency" for a polychromatic one, provided that the source is close to the crystal. This corresponds to the fact that only a small portion of the crystal diffracts in case of a line emission, whereas the whole volume is efficient when using a continuum source.

The above expressions are useful to estimate the expected diffracted intensity for a continuum emission knowing the efficiency measured with a monochromatic source, or vice versa.

\subsection{Beam divergence of the diffracted beam}

Finally consider the effect of the mosaic spread on the divergence of the beam. The mosaic spread of a crystal increases its energy bandwidth and, consequently, the astrophysical interest of a Laue lens. However the mosaicity also induces a divergence of the diffracted beam in the meridian plane. So, since the deviation angle is twice the Bragg angle, the divergence of the beam is twice the angular width of the diffraction curve as given by equation 40. In other words, due to the "reflection" effect on the crystal planes, the divergence of the diffracted beam is roughly twice the mosaic spread of the crystal.

To first order, the diffraction curve is proportional to the crystallites distribution (i.e. a Gaussian whose FWHM is the mosaicity $m$ ). Consequently, in case of an extended crystal and assuming a parallel incoming beam, the radial spot size is then roughly proportional to the convolution of the crystal footprint $(2 \Delta r)$ by a normal distribution, whose FWHM is $2 F_{\text {det }} m, F_{\text {det }}$ being the distance from the lens to the detector. Mathematically, this means for the radial intensity on the detector plane :

$$
I_{\text {det }}(r) \propto \frac{1}{2}\left(\operatorname{erf}\left(\frac{\Delta r-r}{\sqrt{2} \beta}\right)+\operatorname{erf}\left(\frac{\Delta r+r}{\sqrt{2} \beta}\right)\right),
$$

where $\beta=F_{\text {det }} m / \sqrt{2 \ln (2)}$. When $\beta \ll 2 \Delta r$ (i.e if the detector is close to the crystal), $I_{\text {det }}(r)$ tends to a rectangular shape of width $2 \Delta r$, i.e. the projected footprint of the crystal. On the contrary, if $\beta \gg 2 \Delta r$, the radial distribution is close to a Gaussian with a FWHM of $2 F_{\text {det }} m$. This effect of mosaic defocusing (as introduced by N. Lund in this conference) appears finally as the major limiting factor on the focal length and mosaicity.

\section{Conclusion}

The precedent sections described the basics of X-ray diffraction and their application to X-ray and gamma-ray focusing. Based on the Laue diffraction laws and the Darwin model of mosaic crystals, these theoretical developments demonstrated the interest of this technique for astrophysics. An accompanying article (Halloin, in this volume) describes more precisely the application of these concepts to Laue diffraction lenses, comparing experimental results and Monte-Carlo simulations based on the theory presented here.

From this model, the "best" crystal for a Laue lens appears as a trade-off between the different parameters : 
- The mosaicity enables the energy overlap from one ring to another but causes mosaic defocusing.

- The crystallites' mean size should be as small as possible but its effect is energy-dependent, hard to control in practice and correlated with the mosaicity.

- The thickness of the crystal should be of the order of one half of the mean free path but the optimum is flat enough to allow significant deviation in benefit of, e.g., the mass budget.

- The radial extension of the tiles would ideally be very small so that the footprint be limited by mosaic defocusing. In practice, the consequently high number of tiles to be grown and the technical challenge of their cut and etching will temper this wish.

One should nevertheless notice that the mosaic model, although well studied, understood ... and realized, may not be the "best" for our purpose. Especially, the equilibrium between the diffracted and direct beams as well as the relatively large beam divergence (due to the wings of the crystallites' angular distribution) limit the efficiency of the crystals. Further improvements in crystal growth or "synthesis" may potentially overcome these issues (e.g. see the article of R.K. Smither on gradient crystals in this volume [16]).

To conclude, one should note that a computer program exists that can calculate many of the important crystal diffraction parameters, without the need of knowing the complicated mathematics behind the calculations. This program, called XOP (and available on many websites such as [17]) is extensively used in the synchrotron community and could now also be useful to the astrophysics community...

\section{References}

1. P. K. W. Friedrich and M. Laue, Annalen der Physik 41, 971 (1913).

2. M. Laue, Annalen der Physik 41, 989 (1913).

3. H. Halloin, this volume (2006).

4. M. Gouy, Annales de Physique et de Chimie 5, 241 (1916).

5. R. Dardord, Journal de Physique et Le Radium 3, 218 (1922).

6. E. Fermi, Nuovo Cimento 25, 63 (1923).

7. A. Guinier, Théorie et Technique de la Radiocristallographie (Dunod, Paris, 1956).

8. H. Halloin, Ph.D. thesis, University Paul Sabatier, Toulouse (2003).

9. B. E. Warren, X-ray Diffraction (Addison-Wesley Publishing Company, 1969).

10. W. H. Zachariasen, Theory of X-Ray Diffraction in Crystals (Dover Publications Inc., New York, 1945).

11. W. H. Zachariasen, Acta Crystallographica 16, 1139 (1963).

12. D. T. Cromer and J. T. Waber, in International Tables for X-Ray Crystallography (Kynoch Press, Birmingham, 1974), vol. 4, pp. 71-147.

13. C. G. Darwin, The Philosophical Magazine 27, 315 (1914).

14. C. G. Darwin, The Philosophical Magazine 27, 675 (1914).

15. C. G. Darwin, The Philosophical Magazine 43, 800 (1922). 
16. R. K. Smither, this volume (2006).

17. M. Sanchez del Rio and R. J. Dejus, Web page of the X-ray Oriented Programs (XOP), http://www.esrf.fr/computing/scientific/xop2.1/ (2003). 